\title{
A study of Tycho's SNR at TeV energies with the HEGRA CT-System
}

F. A. Aharonian ${ }^{1}$, A. G. Akhperjanian ${ }^{7}$, J. A. Barrio ${ }^{2,3}$, K. Bernlöhr ${ }^{1}$, H. Börst ${ }^{5}$, H. Bojahr 6 , O. Bolz ${ }^{1}$, J. L. Contreras ${ }^{3}$, J. Cortina ${ }^{2}$, S. Denninghoff ${ }^{2}$, V. Fonseca ${ }^{3}$, J. C. Gonzalez ${ }^{3}$, N. Götting ${ }^{4}$, G. Heinzelmann ${ }^{4}$, G. Hermann ${ }^{1}$, A. Heusler ${ }^{1}$, W. Hofmann ${ }^{1}$, D. Horns ${ }^{4}$, A. Ibarra ${ }^{3}$, I. Jung ${ }^{1}$, R. Kankanyan ${ }^{1,7}$, M. Kestel ${ }^{2}$, J. Kettler ${ }^{1}$, A. Kohnle ${ }^{1}$, A. Konopelko ${ }^{1}$, H. Kornmeyer ${ }^{2}$, D. Kranich ${ }^{2}$,

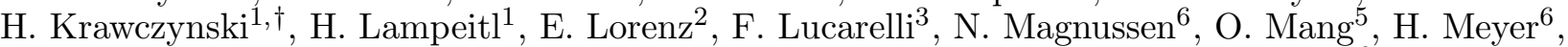
R. Mirzoyan ${ }^{2}$, A. Moralejo ${ }^{3}$, L. Padilla ${ }^{3}$, M. Panter ${ }^{1}$, R. Plaga ${ }^{2}$, A. Plyasheshnikov $^{1, \S}$, J. Prahl $^{4}$, G. Pühlhofer ${ }^{1}$, G. Rauterberg ${ }^{5}$, A. Röhring ${ }^{4}$, W. Rhode ${ }^{6}$, G. P. Rowell ${ }^{1}$, V. Sahakian ${ }^{7}$, M. Samorski ${ }^{5}$, M. Schilling ${ }^{5}$, F. Schröder ${ }^{6}$, W. Stamm ${ }^{5}$, M. Tluczykont ${ }^{4}$, H. J. Völk ${ }^{1}$, C. Wiedner ${ }^{1}$, and W. Wittek ${ }^{2}$

1 Max-Planck-Institut für Kernphysik, Postfach 103980, 69029 Heidelberg, Germany

2 Max-Planck-Institut für Physik, Föhringer Ring 6, 80805 München, Germany

3 Universidad Complutense, Facultad de Ciencias Físicas, Ciudad Universitaria, 28040 Madrid, Spain

4 Universität Hamburg, II. Institut für Experimentalphysik, Luruper Chaussee 149, 22761 Hamburg, Germany

${ }^{5}$ Universität Kiel, Institut für Experimentelle und Angewandte Physik, Leibnizstraße 15-19, 24118 Kiel, Germany

${ }^{6}$ Universität Wuppertal, Fachbereich Physik, Gaußstr.20, 42097 Wuppertal, Germany

7 Yerevan Physics Institute, Alikhanian Br. 2, 375036 Yerevan, Armenia

$\dagger$ Present address: Astronomy Dept., Yale University, PO Box 208101, New Haven, CT 06520-8101, USA

$\S$ On leave from Altai State University, Dimitrov Street 66, 656099 Barnaul, Russia

Received 15 December 2000 / Accepted 24 April 2001

\begin{abstract}
Tycho's supernova remnant (SNR) was observed during 1997 and 1998 with the HEGRA Čerenkov Telescope System in a search for gamma-ray emission at energies above $\sim 1 \mathrm{TeV}$. An analysis of these data, $\sim 65$ hours in total, resulted in no evidence for TeV gamma-ray emission. The $3 \sigma$ upper limit to the gamma-ray flux $(>1 \mathrm{TeV})$ from Tycho is estimated at $5.78 \times 10^{-13}$ photons $\mathrm{cm}^{-2} \mathrm{~s}^{-1}$, or 33 milli-Crab. We interpret our upper limit within the framework of the following scenarios: (1) that the observed hard X-ray tail is due to synchrotron emission. A lower limit on the magnetic field within Tycho may be estimated $B \geq 22 \mu \mathrm{G}$, assuming that the RXTE-detected X-rays were due to synchrotron emission. However, using results from a detailed model of the ASCA emission, a more conservative lower limit $B \geq 6 \mu \mathrm{G}$ is derived. (2) The hadronic model of Drury and (3) the more recent time-dependent kinetic theory of Berezhko \& Völk. Our upper limit lies within the range of predicted values of both hadronic models, according to uncertainties in physical parameters of Tycho, and shock acceleration details. In the latter case, the model was scaled to suit the parameters of Tycho and re-normalised to account for a simplification of the original model. We find that we cannot rule out Tycho as a potential contributor at an average level to the Galactic cosmic-ray flux.
\end{abstract}

Key words. gamma rays: observations - ISM: supernova remnants: individual objects: Tycho's SNR

\section{Introduction}

The search for gamma-ray emission of TeV energies from supernova remnants (SNRs) in recent years is motivated by the need to explain the origin of Galactic cosmic-rays (CR). SNRs are long-believed primarily responsible for the Galactic CR population, matching the CR energetics and spectral index (Völk 1997; Baring 2000a). The production of $\gamma$-rays in SNRs is thought to result from the

Send offprint requests to: G. P. Rowell,

e-mail: Gavin.Rowell@mpi-hd.mpg.de interaction of shock-accelerated particles (hadrons and electrons) with the interstellar medium (ISM) and local soft photon fields. Being relatively unattenuated over long distances and preserving the production site directionality, gamma radiation is one of the most accessible tracers of CR acceleration in the universe.

$\mathrm{TeV}$ gamma radiation is primarily expected from two channels (1) Collisions of hadronic CRs, producing gamma-rays via $\pi^{\circ}$ decay and (2) CR electrons upscattering soft photons via the inverse Compton (IC) process, and CR electron collisions via Bremsstrahlung. 
Detailed modelling of SNR environments has revealed distinct spectral features in the $\mathrm{GeV} / \mathrm{TeV}$ regime for these processes, and in combination with radio and $\mathrm{X}$-ray observations, those in the $\mathrm{TeV}$ regime are deemed vital in establishing SNRs as sites of CR production (Drury et al. 1994; Naito \& Takahara 1994; Berezhko \& Völk 1997; Baring et al. 2000b).

So far, evidence for $\mathrm{TeV}$ emission has come from CANGAROO observations of two southern hemisphere SNRs, SN 1006, and SNR RX J1713.7-3946 (Tanimori et al. 1998; Muraishi et al. 2000), and the HEGRA Čerenkov Telescope CT-System (High Energy Gamma Ray Astronomy Čerenkov Telescope) after deep observations of Cas-A (Pühlhofer et al. 1999; Aharonian et al. 2000a). Upper limits are reported for those other promising SNR candidates observed so far in both the TeV and PeV regimes (Buckley et al. 1998; Prosch et al. 1996; Goret et al. 1999; Allen et al. 1995; Rowell et al. 2000), including Tycho's SNR, for which the Whipple Collaboration obtained 14.5 hours data. The CANGAROO and HEGRA detections might be interpreted in the framework of the SNR as a source of multi-TeV CR electrons by virtue of a strong non-thermal tail in their X-ray spectra above $1 \mathrm{keV}$ (Koyama et al. 1995, 1997; Allen et al. 1995). On the other hand as argued by Aharonian \& Atoyan (1999) and Berezkho et al. (1999) for SN 1006, and by Atoyan et al. (2000) for Cas-A, the $\mathrm{TeV}$ results do not rule out the hadron channel. It is nevertheless less clear at the moment as to the location of CR hadron accelerators in our galaxy.

Tycho's SNR (G120.1+1.4, 3C 10) is one of the most intensely studied SNRs. It is an archetypal shell-type (radio \& X-Ray) SNR, formed most likely from a type Ia supernova (SN 1572), and has expanded at $\sim 0.1 \% \mathrm{yr}^{-1}$ to a radius $\sim 4^{\prime}$ (Katz-Stone et al. 2000 and references therein). One estimate of distance is put at $2.2 \mathrm{kpc}$ (Albinson et al. 1986 and references therein) based on proper motion studies, absorption against field stars and the fact that Tycho appears embedded in the Perseus arm of the Milky Way. A higher distance estimate of $4.5 \mathrm{kpc}$ (Schwarz et al. 1995) is derived from a model of the HI spectrum and number of $\mathrm{HI}$ absorbing features in the region. The radio synchrotron emission $(20 \& 90 \mathrm{~cm})$ shows variation in photon index that appears correlated with edge filaments, perhaps tracing regions of enhanced magnetic field and particle acceleration (Katz-Stone et al. 2000). Studies at the HI 21 $\mathrm{cm}$ line indicate expansion on the eastern side of Tycho is slowed by a region of higher density $\left(160-325 \mathrm{~cm}^{-3}\right.$, Reynoso et al. 1999), suggesting that Tycho's SNR may not be expanding into such a homogeneous region as earlier believed. The X-ray emission shows very strong line features and overall, is well fit by thermal bremsstrahlung components (Fink et al. 1994; Petre et al. 1999). A power law however is necessary to fit a hard X-ray tail. This presumably non-thermal tail above $1 \mathrm{keV}$ suggests that Tycho may a source of electrons up to $\sim 100 \mathrm{TeV}$, thereby joining the growing number of SNRs that exhibit this feature (see Petre et al. 1999 for a review).
Table 1. Summary of Tycho Observations by the HEGRA CT-System. The CR event rate is after the size cut of 40 photoelectrons and the CR rate in brackets for 1997 is prior to a cut of 10 photoelectrons on second brightest image pixel.

\begin{tabular}{lrrr}
\hline \hline & 1997 & 1998 & Total \\
\hline Obs. time (hrs) & 19.7 & 44.9 & 64.6 \\
Selected runs & 50 & 115 & $165(82 \%)$ \\
Configuration & $\mathrm{CT} 3-6$ & $\mathrm{CT} 3-6$ & \\
Zenith angle range & $32-41^{\circ}$ & $32-45^{\circ}$ & \\
CR Event Rate (Hz) & $8.4(10.3)$ & 8.5 & 8.5 \\
\hline \hline
\end{tabular}

\section{Observations}

The observations of Tycho's SNR were made using the HEGRA CT-System incorporating, at the time of data taking (1997 \& 1998), four identical imaging air Čerenkov telescopes ${ }^{1}$ (CT3-6). The telescopes are operated in coincidence to achieve a stereoscopic view of Čerenkov light air showers induced by $\gamma$ and CR primaries. The CT-System is situated on the Roque de los Muchachos at La Palma (2200 $\mathrm{m}$ asl, $28^{\circ} 45^{\prime} \mathrm{N} 17^{\circ} 54^{\prime} \mathrm{W}$ ) and at the zenith angles of these data, operates at an energy threshold for $\gamma$-ray primaries of $\sim 1 \mathrm{TeV}$ (see Konopelko et al. 1999 and references therein).

The Tycho data were accumulated in Jul.-Sep. 1997 and Aug.-Dec. 1998 in 20-30 min runs utilising the wobble mode of observation. In this mode, the source is offset $\pm 0.5^{\circ}$ in declination in alternating runs, enabling the background or OFF source data to be estimated from the opposing position (or a series of positions) $1.0^{\circ}$ away. Table 1 gives a summary of these observations.

Approximately $20 \%$ of all runs were rejected, due primarily to weather effects. Bad runs were identified according to the criteria that the image width from each triggered telescope and stereo trigger rate match their expected values accordingly, within $6 \%$ for width and $15 \%$ for rate (representing levels $<4 \sigma$ outside their Gaussian distribution means). The bad runs actually comprised the non-Gaussian tails of these distributions. The trigger rate for CRs was significantly lower in 1998 compared to 1997 (by $\sim 25 \%$ ), attributed mainly to photomultiplier tube (PMT) fatigue and mirror degradation. We accounted for this threshold difference by artifically raising the trigger threshold of 1997 data to 1998 levels by applying a 10 photoelectron threshold to the second-brightest pixel in the image, mimicking the hardware trigger threshold, at 8 photoelectrons. The effect of this software trigger is to simplify analysis and in particular, the estimation of upper limits. It may be justified given the negligible effect on the $\gamma$-ray threshold of this cut (following the 40 photoelectron cut described below) and the fact that the 1997 observation time is $\sim 45 \%$ that of 1998 . The $\gamma$-ray energy threshold ${ }^{2}$ for both years' data were found from

\footnotetext{
1 Since 1999, the full five telescopes have been in operation, CT2 being the most recent telescope brought online.

${ }^{2}$ The maximum differential trigger rate, for a number of photon indices.
} 
simulations to be consistent within systematic errors $(15 \%)$ to a value of $1 \mathrm{TeV}$.

Event filtering follows the standard method in that images containing more than 40 photoelectrons (size) are selected for further analysis. The rejection of the CR background is achieved with cuts on the image shape and the location of its reconstructed source position. The reconstruction is described as "Algorithm 1" by Hofmann et al. (1999) and the shape cut used is the so-called mean scaled width (msw). The msw is the average of the scaled widths from each accepted image. Each image is scaled according to it's expected value ( $\gamma$-ray hypothesis), dependent upon image size, zenith angle of observation and impact distance of the event from the CT-System centre. Following cuts on $m s w$ and $\theta$, the difference between the reconstructed and actual source positions, the stereoscopic method permits unprecedented angular and energy resolution for $\gamma$-ray images on an event-by-event basis of $\sim 6^{\prime}$ and $\sim 20 \%$ respectively (Aharonian et al. 2000b).

We use a combination of "tight cuts" in the shape $(0.4<m s w<1.1)$ and direction $\left(\theta^{2}<0.02 \mathrm{deg}^{2}\right)$ criteria to preferentially select $\gamma$-ray images against those from CR. Tight cuts are optimal in the search for weak signals in background dominated statistics (3-5 $\sigma$ level), with the caveat that systematic effects may become more important due to the cuts lying close to distribution tails. Tycho is a marginally extended source for the HEGRA CT-System, and thus the optimal $\theta^{2}$ cut will be slightly larger than that for a point source at $\sim 0.015 \mathrm{deg}^{2}$. A simple Monte Carlo, matching roughly the statistics of the background of our Tycho dataset, was used to determine an optimal $\theta^{2}$ cut, assuming various morphologies for the SNR with the results displayed in Fig. 1. Given the uncertainty in the SNR morphology (disk, Gaussian, annular or a combination), a $\theta^{2}$ cut of $0.02 \mathrm{deg}^{2}$ was deemed suitable.

A useful indicator of data quality is the CR acceptance $\left(\kappa_{\mathrm{CR}}\right)$ of the msw cut on a run-by-run basis (Fig. 2 ). We can see that $\kappa_{\mathrm{CR}}$ is consistent over the entire dataset and moreover, the cut of 10 p.e on the second brightest pixel applied to 1997 data introduced no significant change in $\kappa_{\mathrm{CR}}$.

\section{Results}

We evaluate the statistical significance of the post-cut excess using Eq. (9) of Li \& Ma (1983). The background or OFF source counts are taken from three control regions at position angles $180^{\circ} \& \pm 90^{\circ}$, on a circle $0.5^{\circ}$ radius centred on the tracking position, as the wobble mode of observation permits. A normalisation factor of $\frac{1}{3}$ is therefore used in the significance calculation. By using more than one background region, effects of skynoise, camera response and zenith angle difference are averaged out further (on top of that already afforded by the stereoscopic technique), reducing somewhat any systematics expected from such properties, and moreover, reducing the statistical error in the background. A distribution of $\theta^{2}$ for the combined data after application of the msw cut

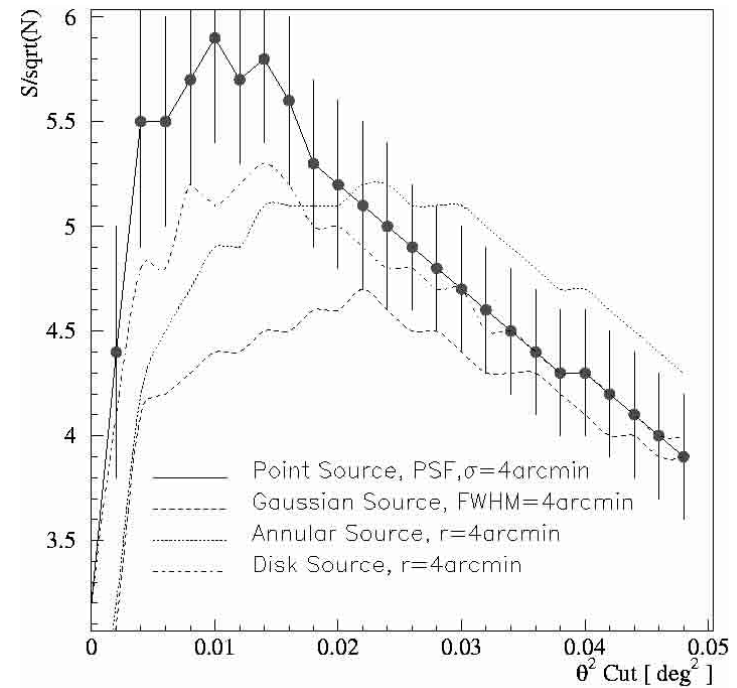

Fig. 1. Signal to noise ratios (unnormalised curves) from a Monte Carlo for sources of various morphologies. The HEGRA CT-System point spread function (PSF, with $\sigma$ specified in the plot) is assumed to be a Gaussian and based on measurements on the Crab (Aharonian et al. 2000d). Here 100000 background events were sampled, reproducing roughly the statistics of the Tycho dataset, and the number of source events chosen to reproduce $\mathrm{a} \sim 5 \sigma$ maximum excess.

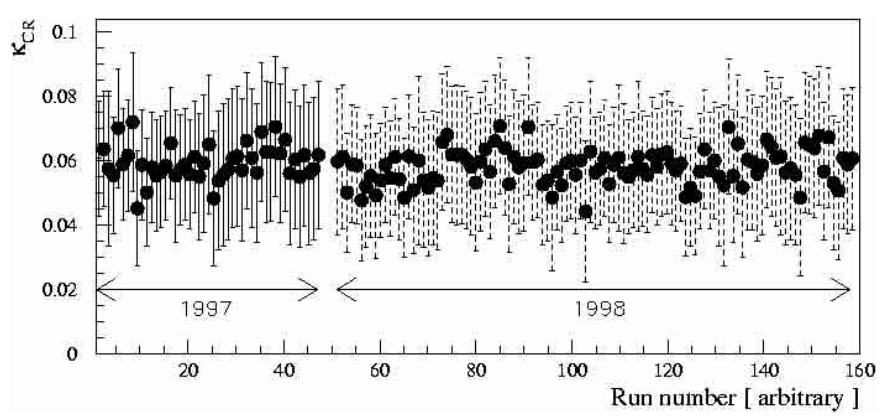

Fig. 2. Cosmic ray acceptance $\left(\kappa_{\mathrm{CR}}\right)$ of the msw cut on a runby-run basis. The 1997 data were also subjected to a cut on the second-brightest pixel of 10 p.e.

Table 2. Post-cut (after $m s w \& \theta^{2}$ cuts) statistics of the Tycho data (significance calculated using Eq. (9) of Li \& Ma 1983) taking as the background, the sum of positions $b_{i}$. The $3 \sigma$ upper limit (99.7\% UL) is expressed in Crab and absolute units (see text).

\begin{tabular}{lrrr}
\hline \hline & 1997 & 1998 & Total \\
\hline ON & 127 & 259 & 386 \\
OFF $\left(\Sigma_{i}^{3} b_{i}\right)$ & 383 & 871 & 1254 \\
ON-OFF $(\sigma)$ & -0.7 & -1.6 & -1.4 \\
$3 \sigma$ UL $(\mathrm{Crab})$ & & & 0.033 \\
$3 \sigma$ UL $\left(F(>1 \mathrm{TeV}) \times 10^{-13} \mathrm{ph} \mathrm{cm}^{-2} \mathrm{~s}^{-1}\right)$ & 5.78 \\
\hline \hline
\end{tabular}

discussed above is presented in Fig. 3. We summarise further the results in Table 2. No significant excess was seen in either dataset, and the combined excess represents a significance of $-1.4 \sigma$. We may express our upper limit in absolute units by using the $\mathrm{TeV}$ flux from the 


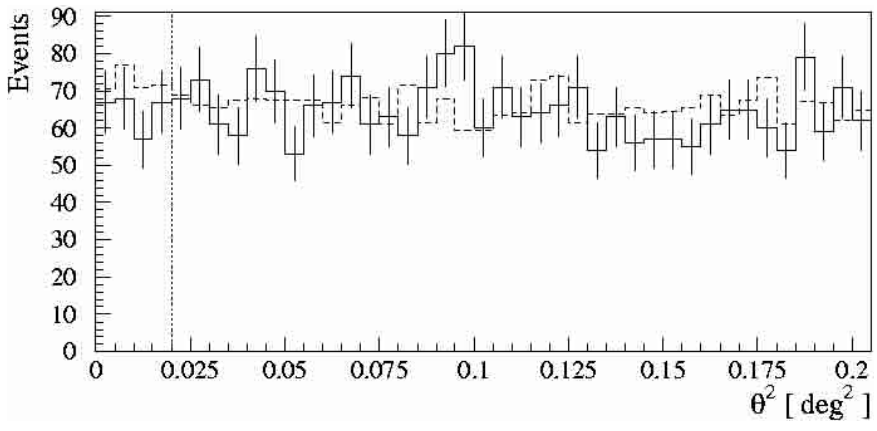

Fig. 3. Distribution of $\theta^{2}$ for combined 1997 and 1998 observations of Tycho's SNR (ON source, solid line). The OFF source data (dashed line) are taken from three positions in the field of view (see text) and scaled by a factor $\frac{1}{3}$. The optimal cut is indicated by the dotted vertical line.

Crab Nebula, thereby taking advantage of the substantial simulation effort in deriving the absolute Crab flux (Aharonian et al. 2000b). Fluctuating the ON-OFF excess according to Helene (1983) and using the method described by Aharonian et al. (2000c), our $3 \sigma$ UL (99.7\%) corresponds to 33 milli-Crab ${ }^{3}$. Here, those Crab data used to estimate the UL $(\sim 6$ hours in total), were subjected to an identical analysis as described above, and selected to match the zenith angle distribution as that for Tycho. Above the threshold energy of $1 \mathrm{TeV}$, our UL is $F(>1 \mathrm{TeV})=5.78 \times 10^{-13} \mathrm{ph} \mathrm{cm}^{-2} \mathrm{~s}^{-1}$.

Finally, we should point out that the method of Aharonian et al. (2000c) (which downward fluctuates the Crab excess) to estimate the UL in Crab units was tested for accuracy with a simple Monte Carlo and found to overestimate a true $3 \sigma$ UL (for the numbers of Table 2 by about $25 \%$. This overestimate is effectively canceled however by the negative bias of our $-1.4 \sigma$ excess, an underestimate of $\sim 30 \%$ relative to a zero excess result. We therefore may assume that our $3 \sigma$ UL represents closely a true estimate. Secondly, any integral UL or flux estimate quoted in absolute units will naturally depend somewhat on the difference in photon index between the Crab and Tycho. This uncertainty amounts to $\leq 30 \%$ on the UL estimation for a $20 \%$ uncertainty in the values of $1 \mathrm{TeV}$ and -1.59 respectively for energy and photon index.

\section{Inverse Compton interpretation}

The evidence for a non-thermal tail (Fink et al. 1994, GINGA data; Petre et al. 1999, RXTE data) may suggest that Tycho's SNR is an accelerator of CR electrons to multi-TeV energies. In such a framework, the synchrotron process is assumed to account for these X-ray photons, and the possibility of inverse Compton (IC) $\mathrm{TeV} \gamma$-ray emission should be considered. However, one must be careful here since a power law fit is reasonable only at energies above $\sim 10 \mathrm{keV}$ and, alternative physical explanations to

31 Crab:

$F(>E \mathrm{TeV})=1.75 \times 10^{-11}\left(\frac{E}{1 \mathrm{TeV}}\right)^{-1.59} \mathrm{ph} \mathrm{cm}^{-2} \mathrm{~s}^{-1}$

(Aharonian et al. 2000b). the synchrotron scenario do exist (see for e.g. Asvarov et al. 1990; Laming 1998; Tatischeff et al. 1998).

Assuming that the synchrotron model is valid for Tycho, we may use the direct relationship between the expected IC $\left(f_{\gamma}\right)$ and X-ray energy fluxes ${ }^{4}\left(f_{\mathrm{x}}\right)$ arising from the same electrons:

$\frac{f_{\mathrm{x}}(\epsilon \mathrm{keV})}{f_{\gamma}(E \mathrm{TeV})} \sim 10\left(\frac{B}{10^{-5} \mathrm{G}}\right)^{2}$

to establish a condition on the magnetic field $B$ of the SNR. The mathematical caveat here is that the correct energy range in the $\mathrm{TeV}$ and $\mathrm{keV}$ regimes must be adhered to and that we assume that the emission regions of both components have the same size. In the $\delta$-function approximation of the synchrotron emissivity for an electron, the IC $(E / 1 \mathrm{TeV})$ and X-ray $(\epsilon / 1 \mathrm{keV})$ synchrotron energies in Eq. (1) are coupled according to (see Aharonian et al. 1997):

$\epsilon \sim 0.07\left(\frac{E}{1 \mathrm{TeV}}\right)\left(\frac{B}{10^{-5} \mathrm{G}}\right) \mathrm{keV}$

so that for IC fluxes at $\sim 1 \mathrm{TeV}$, a comparison with the $\mathrm{X}$-ray flux at energies $\sim 0.1$ to $1.0 \mathrm{keV}$ is required for values of $B \sim 10$ to $100 \mu \mathrm{G}$ expected in a SNR. Equation (1) follows from the fact that the ratio of the synchrotron and IC energy fluxes is proportional to the ratio of the energy densities in the magnetic and CMB fields respectively. The dominant IC flux arises from the up-scattering of CMB photons by CR electrons of energy 10 to $100 \mathrm{TeV}$. Preliminary results from RXTE data (Petre et al. 1999) indicate a normalisation (at $1 \mathrm{keV}$ ) and photon index respectively of $0.30 \pm 0.02$ photons $\mathrm{cm}^{-2} \mathrm{~s}^{-1}$ and $-3.18 \pm 0.02$, valid at energies from 10 to $20 \mathrm{keV}$. From earlier GINGA data Fink et al. (1994) derived a power law fit (in combination with a thermal bremsstrahlung and Fe line component) of photon index -2.72 (see Fink for error details) and normalisation of $7.4_{-7.4}^{+8.9} \times 10^{-2} \mathrm{ph} \mathrm{cm}^{-2} \mathrm{~s}^{-1}$ (at $1 \mathrm{keV}$ ), which is valid from 4.5 to $20 \mathrm{keV}$. A direct extrapolation to energies required for the $B$ field estimate, namely 0.1 to $1.0 \mathrm{keV}$, will lead to overestimation of the synchrotron flux since one would expect a turnover near this range. A more reasonable method to estimate fluxes at such lower energies is to fit to the X-ray flux a power law spectrum with exponential cutoff. The RXTE data is preferred for such a fit due to the much reduced errors compared to those of GINGA data. We fit the spectrum using the function:

$E^{2} F(E)=A E^{1-\alpha} \exp \left[-\left(E / E_{\mathrm{m}}\right)^{0.5}\right] \operatorname{ergcm}^{-2} \mathrm{~s}^{-1}$

where $\alpha$ (radio photon index) and $E_{\mathrm{m}}$ (cutoff energy) are free parameters and $A$ is the normalisation determined from the flux density at $1 \mathrm{GHz} S_{1 \mathrm{GHz}}=56 \mathrm{Jy}$ (Green $2000)$. Equation (3) is derived for an electron spectrum with exponential cutoff using the $\delta$-function approximation for the electron synchrotron emissivity and is shown

\footnotetext{
${ }^{4}$ Energy flux: $E^{2} F(E)$.
} 
to adequately describe the X-ray spectrum out to energies $\sim 10 E_{\mathrm{m}}$ (Reynolds 1999). Our fit yields values of $\alpha=0.7$ and $E_{\mathrm{m}}=1.6 \mathrm{keV}$ respectively with $\alpha$ differing somewhat from the radio photon index, listed as 0.61 in Green's catalogue. Katz-Stone et al. (2000) summarise historical measurements of the radio index as varying between 0.5 and 0.7 at different radio bands. From their VLA study, KatzStone indicate an average index of the filaments at 0.52. Results are plotted in Fig. 4a showing our best fit, and that when fixing $\alpha=0.61$. We note that Hwang et al. (1998) have placed an upper limit to the non-thermal luminosity in the 0.5 to $10 \mathrm{keV}$ regime on the order of $\sim 10^{34} \mathrm{erg} \mathrm{s}^{-1}$ ( 0.5 to $10 \mathrm{keV}$ ), based on careful modelling of the nonthermal ASCA detection. In order to accommodate their upper limit to the non-thermal luminosity (for a distance of $2.3 \mathrm{kpc}$ as used by Hwang) our synchrotron spectrum fit to RXTE data must be scaled down by a factor $\sim 15.5$, assuming that their upper limit is exactly $10^{34} \mathrm{erg} \mathrm{s}^{-1}$ (dotted line in Fig. 4a).

If we take the estimated synchrotron fluxes from 0.1 to $1 \mathrm{keV}$ from the direct RXTE fit with our upper limit at $1 \mathrm{TeV}$ for the IC flux, a range of $B_{-5}$ values 22 to $24 \mu \mathrm{G}$, plotted in Fig. 4b (solid line), are obtained, which are reasonably consistent with that expected after amplification by the SNR shock. Using however the lower synchrotron energetics implied by Hwang et al. (1998) we arrive at more conservative lower limits on $B_{-5}$ at $\sim 6 \mu \mathrm{G}$, (dotted line). Such a value would result if little or no amplification was present. Allowing for uncertainty of one order of magnitude in the Hwang estimate (i.e. $5 \times 10^{-33}$ to $4 \times 10^{-34} \mathrm{erg} \mathrm{s}^{-1}$ ) would, for the upper bound case, give a $B$ field lower limit a factor $\sqrt{4}$ higher, i.e. $\sim 12 \mu \mathrm{G}$.

\section{Comparisons with DAV}

We will consider here the production of $\gamma$-radiation by the hadronic or $\pi^{\circ}$-decay channel described in two-fluid model by Drury et al. (1994), hereafter DAV, that has been used extensively in the past (see also Naito \& Takahara 1994). The DAV prediction may be scaled simply according to the energy budget of the SNR $E_{\mathrm{sn}}$, density of the upstream target matter $n$, and distance to the remnant $d$ :

$F_{\gamma} \propto \Theta\left(\frac{E_{\mathrm{sn}}}{10^{51} \mathrm{erg}}\right)\left(\frac{n}{1 \mathrm{~cm}^{-3}}\right)\left(\frac{d}{1 \mathrm{kpc}}\right)^{-2}$.

The parameter $\Theta$ represents the fraction of energy available for the acceleration of CR that are ultimately released into the ISM. The mean value of $\Theta$ per SNR in the galaxy is estimated in the range 0.05-0.3 (Berezhko \& Völk 2000), and pertains to an evolutionary state well into the Sedov phase when accelerated particles start to be released into the ISM. We shall adopt the reasonable, and often used value of $\Theta=0.1$, particle spectral index -1.1 (nominal theory), and for purposes of argument that Tycho has progressed well into the Sedov phase.

Figure 5 highlights a comparison of this DAV prediction under limiting parameter-space selections as calculated by Völk (1997) with our present UL, and those of
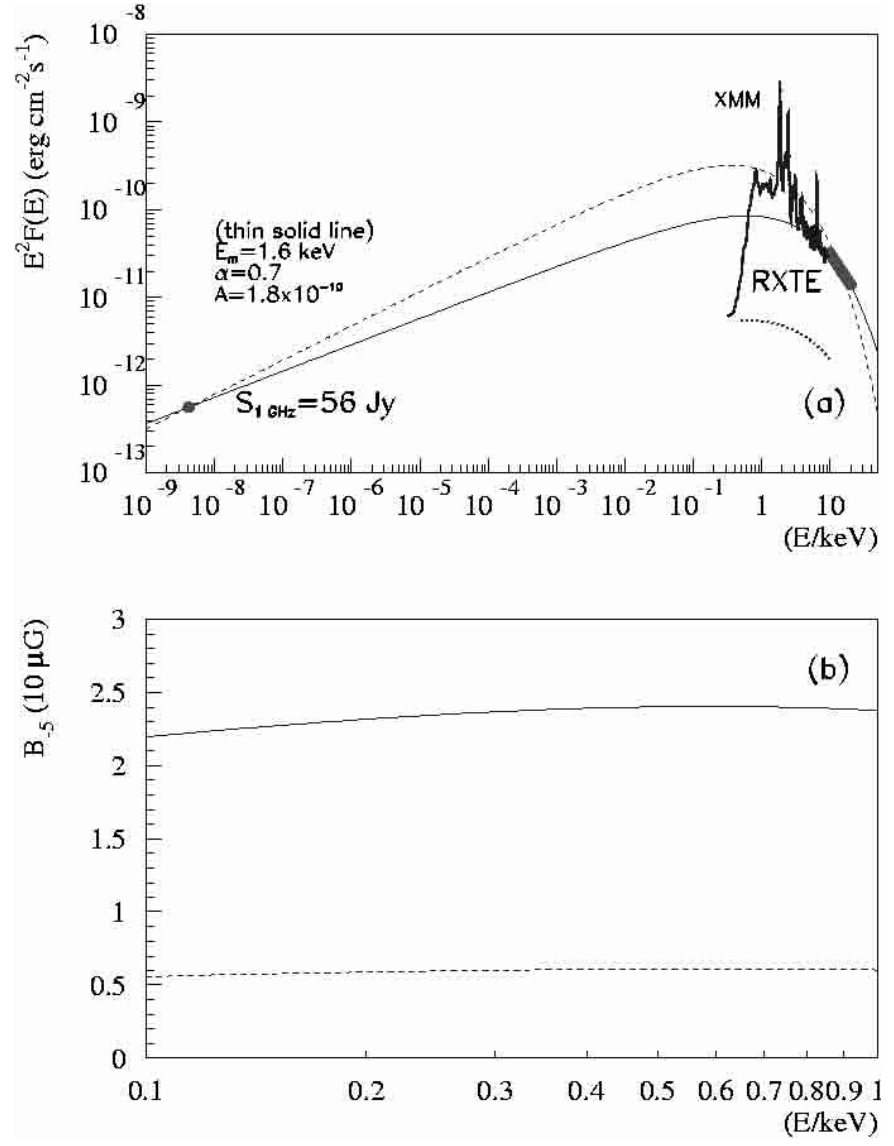

Fig. 4. a) Optimal fit to the RXTE spectrum and radio normalisation using Eq. (3) (thin solid line). (dashed line) A fit when fixing $\alpha=0.61$. (thick solid line) For comparative purposes a model fit to the XMM MOS 1 spectrum from Decourchelle et al. (2001) Fig. 1 is included. Since interstellar absorption is not removed in this fit, the XMM flux below $1.0 \mathrm{keV}$ is therefore a lower limit. (dotted line) Re-scaled RXTE fit to accommodate an upper limit to the non-thermal flux of Hwang et al. (1998). b) (Solid line) Calculation of $B_{-5}(10 \mu \mathrm{G})$ of Eq. (1) taking as $f_{\mathrm{x}}$ the fit given by the solid line of $\mathbf{a}$ ), the RXTE fit, and (dot-dashed line) $B_{-5}$ values obtained when using the scaled-down fit to a synchrotron spectrum given by the dotted line of a) as suggested by Hwang et al. (1998).

previous measurements (Whipple et al. 1998 \& HEGRA AIROBICC, Prahl et al. 1997, and CASA-MIA, Borione et al. 1995).

It is clear that with a factor $\sim 4$ reduction between the Whipple and present upper limits (assuming a spectral index of -1.1 for this comparison), the DAV model is now constrained when allowing for a reasonable range of input parameters. We are approaching the conservative boundary assumed by Völk (1997) for the Sedov phase.

An important parameter in view of the expected $\mathrm{TeV}$ emission from a SNR is it's evolutionary phase. Generally accepted is the notion that the maximum $\mathrm{TeV} \gamma$-ray emission occurs at the beginning of the Sedov phase and a broad maximum in the total CR energy is reached during the Sedov phase. At the former time the mass of swept-up material will just exceed that ejected, and the outer SNR 


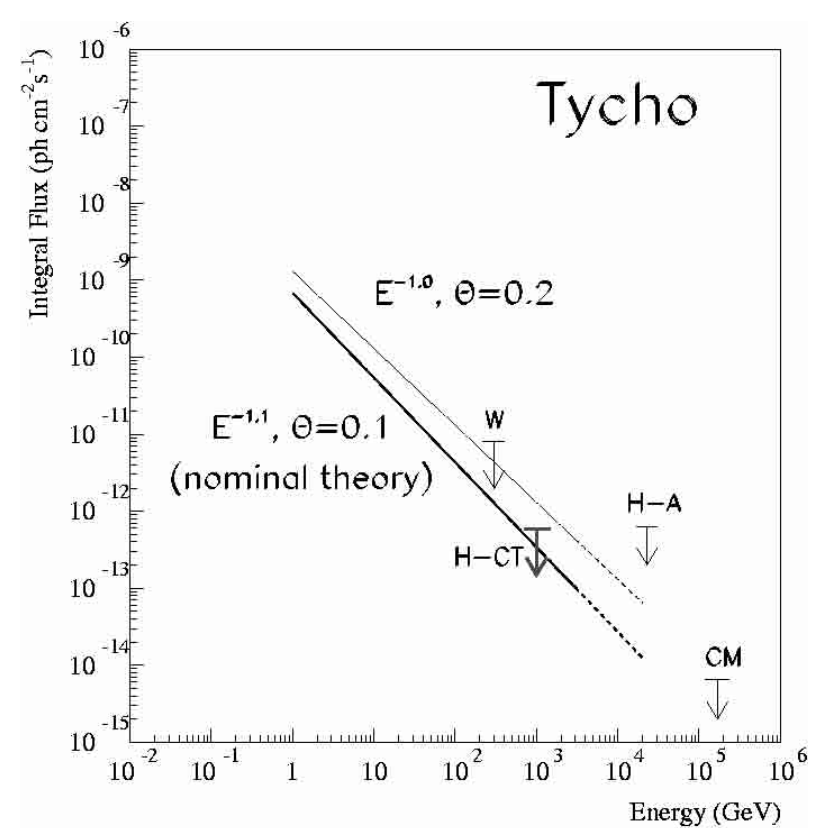

Fig. 5. Upper limits from the present work $(\mathrm{H}-\mathrm{CT})$, and previous measurements (W - Whipple, H-A - HEGRA AIROBICC, CM - CASA-MIA. See text for references) with predictions of the $\pi^{\circ}$-decay $\gamma$-ray flux from the DAV model. These DAV predictions from Völk (1997), use two limiting choices of parameters for $\Theta$, and the spectral index (indicated on the plot). Values for the other parameters were $E_{\mathrm{sn}}=2 \times 10^{50} \mathrm{erg}$ (Smith et al. 1988), $n=1.0 \mathrm{~cm}^{-3}$ and $d=2.3 \mathrm{kpc}$ (Heavens 1984). Note that a reduction of the "nominal theory" curve with a value $\theta=0.05$ may be argued in on the grounds that Tycho is pre-Sedov (see text).

shock begins to expand with radius $R \sim t^{2 / 5}$. Typical ages of Galactic SNRs for the onset of the Sedov phase are 500 to $1000 \mathrm{yrs}$. Tycho is a rather young SNR (428 yrs old) expanding into an ISM with density typical of that expected within the Galactic plane and so it may not be clear as to exactly what phase Tycho is presently in. Observational evidence indicates that globally, Tycho's SNR is near to the Sedov phase $\left(R \sim t^{\nu}, \nu=0.46 \pm 0.02\right.$, Tan \& Gull 1985), and is expanding into, on a global scale at least, a homogeneous ISM. Reynoso et al. (1997) report a similar value, from VLA data for the global expansion, but note that $\nu$ varies between 0.2 and 0.8 around the shell and suggest the presence of denser material along the eastern side (see also recent $21 \mathrm{~cm}$ observations by Reynoso et al. 1999). The expansion rate at X-ray energies is significantly higher than at radio energies, but as in the radio, it also varies considerably azimuthally (Hughes 2000). In contrast to the radio behaviour however, the expansion rate at X-ray energies varies radially, supporting the idea of an ejecta-dominated, or pre-Sedov evolutionary state. Taking these results together with the Rayleigh-Taylor instabilities along the eastern side suggested by Velazquez et al. (1998), we may conclude that the global evolution state of Tycho's SNR is pre-Sedov, although at regions of high density ISM, the evolutionary state will be locally advanced.
A comparison with DAV predictions should therefore allow for the current evolutionary state of Tycho. The lower curve of Fig. 5 (nominal theory) may be lowered further since the age of Tycho is likely less than the sweep-up time $\left(t_{0}=555 \mathrm{yrs}\right)$ which signals the onset of the Sedov phase. A reduction in the $\gamma$-ray emissivity for pre-Sedov epochs can be manifested as a reduction in the $\theta$ parameter to values $<0.1$. A quantitative estimate for $\theta$ in this case is not trivial but generally one can expect a reduction by a factor $\sim 2$ based on consideration of results in Figs. 1 to 3 of DAV, describing different rates of luminosity increase with time. In the next section comparisons to a model dealing with time-dependence in detail are made.

\section{Comparisons with kinetic theory}

The most recent nonlinear kinetic models are the timedependent, spherically symmetric solution of Berezhko \& Völk (1997), hereafter BV, and the Monte Carlo simulation of a quasistationary outer SNR shock in plane parallel geometry by Baring et al. (1999). The BV model is based on the numerical solution of Berezhko et al. (1994) and invokes a distribution of ejecta velocities (e.g. Chevalier \& Liang 1989 and references therein) that contains very high speed components compared to the mean ejecta velocity. At early evolutionary phases this leads to much higher shock speeds than implied by the mean ejecta velocity, and thus to much more intensive CR and $\gamma$-ray production. We will in this paper make use of BV as it was calculated in their paper, scaling the parameters to those of Tycho's SNR. As we shall note shortly, in addition, a physical re-normalisation of these results is necessary. For a more complete treatment, we refer to a companion paper elsewhere in which the BV model will be calculated assuming Tycho parameters.

A scaling of the original BV calculations is required since the start-up parameters (in particular ejected mass $M_{\mathrm{ej}}=10 M_{\odot}$ and SNR energy $E_{\mathrm{sn}}=10^{51} \mathrm{erg}$ ) may differ from those one might expect for Tycho's SNR. For this scaling we may reasonably assume, that the ejecta mass only effects the initial normalisation in the form of the sweep-up time $t_{0}$, and that other parameters will scale the flux in the same way they do the DAV prediction, i.e. independent of time. The first row of Table 3 presents a numerical comparison of our upper limit with the predictions of the BV model, $F_{-13}^{\gamma}$ (in units of $10^{-13} \mathrm{ph} \mathrm{cm}^{-2} \mathrm{~s}^{-1}$ ), scaled to Tycho parameters. $\Theta_{\mathrm{s}}$ is the released fraction of energy available for CRs and $\Theta=0.1$ is the value assumed for an average SNR, discussed previously. Inspection of $F_{-13}^{\gamma}$ for all cases in Table 3 reveals that they are inconsistent with our upper limit.

However, we must now invoke a further, physical renormalisation of the Tycho-scaled BV predictions for the following reason. The BV model assumes a parallel shock geometry (shock normal parallel to the average up-stream $B$ field) over the entire spherical shock surface. For spherical geometry expected of a SNR, such an assumption will only apply for a limited part of the SNR shock 
Table 3. Theoretical $\gamma$-ray fluxes, $F_{-13}^{\gamma}\left(10^{-13} \mathrm{ph} \mathrm{cm}^{-2} \mathrm{~s}^{-1}\right)$ and released relative $\mathrm{CR}$ energies $\Theta_{\mathrm{s}}$ from the BV model scaled according to parameters of Tycho's SNR (see text). Each case represents a choice of injection rate $\eta$ and $B$-field (labeled in the caption of Fig. 6). The parameters subscripted with $r$ refer to those additionally re-normalised to account for the effect of assuming in the BV model, a shock normal parallel to the B field (see results in Fig. 6). The re-normalisation factor here is $(\Theta=0.1) / \Theta_{\mathrm{s}}$, and $\Theta_{\mathrm{r}}(t=428)$ is the relative CR energy calculated at the current age of Tycho's SNR.

\begin{tabular}{lrrrr}
\hline \hline Case & $a$ & $b$ & $c$ & $d^{\dagger}$ \\
\hline$B(\mu \mathrm{G})$ & 5 & 30 & 5 & 5 \\
$\eta$ & $10^{-3}$ & $10^{-3}$ & $10^{-4}$ & $10^{-4}$ \\
\hline$F_{-13}^{\gamma}$ & 91.00 & 49.20 & 20.00 & 9.00 \\
$\Theta_{\mathrm{s}}$ & 0.60 & 0.55 & 0.50 & 0.50 \\
$\Theta_{-} \Theta_{\mathrm{s}}$ & 0.17 & 0.18 & 0.20 & 0.20 \\
$F_{-13}^{\gamma, \mathrm{r}}$ & 15.50 & 9.00 & 4.00 & 1.80 \\
$F_{-13}^{\gamma, \mathrm{r}} / \mathrm{UL}$ & 2.60 & 1.50 & 0.69 & 0.31 \\
$\Theta_{\mathrm{r}}(t=428)$ & 0.06 & 0.04 & 0.04 & 0.04 \\
\hline \multicolumn{5}{l}{ HEGRA CT-System UL $=5.78\left(10^{-13}{\left.\mathrm{ph} \mathrm{cm}^{-2} \mathrm{~s}^{-1}\right) .}^{\dagger}\right.$} \\
$\dagger$ Using a mean value for ejecta velocities.
\end{tabular}

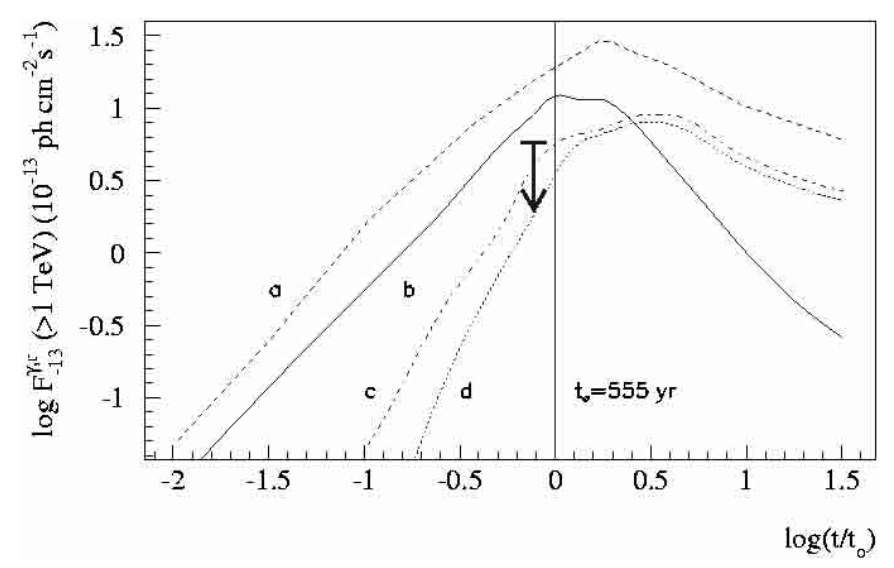

Fig. 6. Time dependence of the $\pi^{\circ}$ decay $\mathrm{TeV} \gamma$-ray integral flux $F_{-13}^{\gamma, \mathrm{r}}\left(10^{-13} \mathrm{~cm}^{-2} \mathrm{~s}^{-1}\right)$ from Tycho for an injection rate $\eta=10^{-3}$ and $B=5 \mu \mathrm{G}$ (dashed line, case a), $\eta=10^{-3}$ and $B=30 \mu \mathrm{G}$ (solid line, case $\mathrm{b}$ ), $\eta=10^{-4}$ and $B=5 \mu \mathrm{G}$ (dash-dotted line, case c). The dotted line (case $\mathrm{d}$ ) represents a less realistic single velocity ejecta case with $\eta=10^{-4}$ and $B=5 \mu \mathrm{G}$, as is adopted in the DAV model. $t_{0}$ is the time of Sedov phase onset. These results have been scaled according to parameters for Tycho $\left(M_{\mathrm{ej}}=1.4 M_{\odot}, E_{\mathrm{sn}}=2 \times 10^{50} \mathrm{erg}\right.$, $n=1.0 \mathrm{~cm}^{-3}, d=2.3 \mathrm{kpc}$. $t_{0}$ is estimated at $555 \mathrm{yrs}$, somewhat larger than the current age of $428 \mathrm{yrs}$ ), and re-normalised by the ratio $\Theta / \Theta_{\mathrm{s}}$. Original model calculations are from Berezhko \& Völk (1997).

surface. Over other parts the shock is quasi-perpendicular. A strongly reduced injection efficiency below that derived from injection theory for parallel shocks $(\eta \sim$ $10^{-3}-10^{-4}$ ) along with a commensurate reduction of the particle production will be noticed in such regions (regarding the question of injection in general, see Kirk \& Dendy 2001 for a recent review). Correcting for this effect in detail is complicated, and will be discussed in a follow-up paper. However, a rough, but empirically-argued implementation of a re-normalisation is achieved by reducing the fluxes $F_{\gamma}^{13}$ by the ratio $\Theta / \Theta_{\mathrm{s}}$ to a re-normalised flux $F_{-13}^{\gamma, \mathrm{r}}=F_{-13}^{\gamma} \Theta / \Theta_{\mathrm{s}}$, where $\Theta=0.1$ is the empirically expected value for an average Galactic SNR discussed earlier (and adopted by DAV), and $\Theta_{\mathrm{s}}$ is that value predicted by the BV model. The re-normalised fluxes are those $r$ subscripted in Table 3, and are rather close to our upper limit and the relative CR energy calculated at the present age of Tycho. $\Theta_{\mathrm{r}}(t)=E_{\mathrm{cr}}(t) / E_{\mathrm{sn}} \times \Theta / \Theta_{\mathrm{s}}$ corresponds to values of around $10^{49}$ ergs. Figure 6 graphically compares $F_{-13}^{\gamma, \mathrm{r}}$ for the different cases discussed. It appears that cases (c) and (d), with a lower injection rate and $B$ field, are preferred. However, the combinations of $B$ and $\eta$ used here are not exhaustive. For example a case assuming higher $B$ (perhaps suggested by our inverse Compton interpretation in Sect. 4) and low injection rate has not been tested by BV. More thorough comparisons will be made later. The uncertainties in scaling parameters, $d, E_{\mathrm{sn}}$ and $n$ will weaken of course the conclusion favouring cases (c) and (d), in which lower injection rates and $B$ fields are assumed. The uncertainty in $d$, where roughly a factor of two above the value used here $(2.3 \mathrm{kpc})$ is published (Schwarz et al. 1995), will have strong influence. Also, a somewhat lower value of $n=0.3 \mathrm{~cm}^{-3}$ derived by Seward et al. (1983) for the pre-shock density may also be preferable to the value given by Smith et al. (1988) used here. At this point we would conclude that a general consistency with the BV model is obtained, perhaps favouring lower injection rates and higher $B$ fields (noting the reduction in emissivity between cases (a) and (b), due to a change in $B$ field), but note that use of a wider parameter space under a dedicated BV calculation is required.

\section{Conclusion}

A search for TeV $\gamma$-radiation from Tycho's SNR has been performed over two years (1997 \& 1998) with the HEGRA CT-system. We find no evidence for such emission and the upper limit ( $3 \sigma$ level) to the $\mathrm{TeV}$ flux is estimated at 33 milli-Crab or $5.78 \times 10^{-13} \mathrm{ph} \mathrm{cm}^{-2} \mathrm{~s}^{-1}$, a value which is a factor $\sim 4$ less than that previously published by the Whipple collaboration (when assuming a spectral index of -1.1 in for the comparison).

Making use of preliminary RXTE results, it is possible to set a lower limit on the magnetic field in Tycho $B \geq 22 \mu \mathrm{G}$ under the assumption that the observed hard or non-thermal tail in the X-ray spectrum is attributed to synchrotron radiation from multi-TeV electrons. A more conservative $B$ field lower limit of $\sim 6 \mu \mathrm{G}$ is obtained if we use the upper limit to the non-thermal X-ray flux estimated by Hwang et al. (1998). It may therefore be premature to draw conclusions about the minimum magnetic field until full analysis of RXTE data are complete. Interestingly the upper limit to the non-thermal energetics of Hwang et al. (1998) is well below that implied by a purely non-thermal interpretation of the RXTE and GINGA results. 
Comparisons to a long-adopted model of $\mathrm{TeV}$ emission from the decay of $\pi^{\circ}$ were made (Drury et al. 1994, denoted DAV), in the initial case assuming that Tycho's SNR is well in to the Sedov phase of evolution. Our upper limit is close to a conservative prediction of DAV. Uncertainty in the global evolutionary state (Sedov, or pre Sedov) of Tycho presents a complication upon comparison to the DAV $\pi^{\circ}$ decay $\gamma$-ray predictions. As a function of time, advanced kinetic theory (Berezhko \& Völk 1997, denoted BV) indicates a relatively early rise in the $\gamma$-ray emissivity of the hadronic channel for pre-Sedov epochs, in contrast to the less realistic case of single-velocity ejecta, as adopted in the DAV model. We find that after adjusting the predictions of BV, firstly scaling for Tycho's parameters and secondly, re-normalising to account for quasiperpendicular shock directions expected in an SNR, a general consistency with our upper limit is found for a range of injection rates and $B$ fields.

We conclude from this non-detection and the interpretation assuming Tycho to be a source of multi- $\mathrm{TeV}$ electrons and hadrons that Tycho's SNR is yet to be ruled out as an average accelerator of Galactic CRs. Our upper limit supports the notion that Tycho is in a pre-Sedov evolutionary state. A more complete investigation will be left to a companion paper in which a dedicated calculation in the framework of the BV model will be performed for the parameters (and their uncertainties) of Tycho's SNR.

Resulting from a type Ia supernova, Tycho's SNR in principle represents the simplest category in terms of a theoretical understanding of the SNR dynamics and interaction with the ISM. Yet, given the complexity in modeling non-thermal processes, the basic consistency between experiment and theoretical predictions certainly encourages future observations of Tycho's SNR and other examples of it's class. Because of the $\sqrt{t}$ dependence on instrument sensitivity, it is likely that further observations at $\mathrm{TeV}$ energies of Tycho will have to wait for the next generation of telescopes operating in the northern hemisphere (e.g. MAGIC, Lorenz et al. 1999; VERITAS, Krennrich et al. 1999). With roughly one order of magnitude improvement in sensitivity for the next generation instruments over that currently available, and the fact that current theory does not leave much room for non-detection at higher instrument sensitivity, a decisive test of the question of whether or not Tycho's SNR contributes to the Galactic CR population at an average level is expected within the next few years.

Acknowledgements. We acknowledge the financial support of the German Ministry for Research and Technology (BMBF) and the Spanish Research Council. We thank the Instituto Astrofísica de Canarias for the use of their site and for the excellent working conditions at La Palma. The technical support staff of the Heidelberg, Kiel, Munich and Yerevan Institutes are also acknowledged. Glenn Allen is thanked for providing a fit to the RXTE Tycho data, and Anne Decourchelle for the model fit to the XMM data. GPR gratefully acknowledges receipt of a von Humboldt Fellowship. An anonymous referee is thanked for helpful comments.

\section{References}

Aharonian, F. A., Atoyan, A. M., \& Kifune, T. 1997, MNRAS, 291, 162

Aharonian, F. A., \& Atoyan, A. M. 1999, A\&A, 351, 330

Aharonian, F. A., Akhperjanian, A. G., Andronache, M., et al. 2000a, A\&A, submitted

Aharonian, F. A., Akhperjanian, A. G., Barrio, J. A., et al. 2000b, ApJ, 539, 317

Aharonian, F. A., Akhperjanian, A. G., Barrio, J. A., et al. 2000c, A\&A, 353, 847

Aharonian, F. A., Akhperjanian, A. G., Barrio, J. A., et al. 2000d, A\&A, 361, 1073

Albinson, J. S., et al. 1986, MNRAS, 219, 427

Allen, G. E., Berley, D., Biller, S., et al. 1995, ApJ, 448, L25

Asvarov, A. I., Dogiel, V. A., Guseinov, O. H., \& Kasumov, F. K. 1990, A\&A, 229, 196

Atoyan, A. M., Aharonian, F. A., Tuffs, R. J., \& Völk, H. J. 2000, A\&A, 355, 211

Baring, M. G. 2000a, in Rapportuer, vol. 26th ICRC, ed. B. L. Dingus (AIP, New York, 2000)

Baring, M. G. 2000b, Towards a Major Atmospheric Cherenkov Detector-VI, ed. B. L. Dingus, M. H. Salamon, \& D. B. Kieda (AIP Conf. Ser. 515, New York, 2000)

Baring, M. G., Ellison, D. C., Reynolds, S. P., et al. 1999, ApJ, 513,311

Barrio, J. A., et al. 1998, The MAGIC Telescope, design study [MPI-PhE/98-5]

Berezhko, E. G., Yelshin, V. K., \& Ksenofontov, L. T. 1994, Astropart. Phys., 2, 215

Berezhko, E. G., \& Völk, H. J. 1997, Astropart. Phys., 7, 183

Berezhko, E. G., Ksenofontov, L. T., \& Petukhov, S. I. 1999, Proc. 26th Int. Cosmic Ray Conf. (Salt Lake City) 4, 431

Berezhko, E. G., \& Völk, H. J. 2000, ApJ, 540, 923

Borione, A., Catanese, M., Covault, C. E., et al. 1995, Proc. 24th Int. Cosmic Ray Conf. (Rome) 2, 439

Buckley, J. H., Akerlof, C. W., Carter-Lewis, D. A., et al. 1998, A\&A, 329, 639

Chevalier, R. A., \& Liang, E. P. 1989, ApJ, 344, 332

Decourchelle, A., Sauvageot, J. L., Audard, M., et al. 2001, A\&A, 365, L218

Drury, L. O'C, Aharonian, F., \& Völk, H. J. 1994, A\&A, 287, 959

Fink, H. H., Asaoka, I., Brinkman, W., et al 1994, A\&A, 283, 635

Goret, P., Gouiffes, C., Nuss, E., et al. 1999, Proc. 26th Int. Cosmic Ray Conf. (Salt Lake City) 3, 496

Green, D. A. 2000, A Catalogue of Galactic Supernova Remnants (2000 August version), Mullard Radio Astronomy Observatory, Cavendish Laboratory, Cambridge, UK (available on the World-Wide-Web at http://www.mrao.cam.ac.uk/surveys/snrs/).

Heavens, A. F. 1984, MNRAS, 211, 195

Helene, O. 1983, Nucl. Inst. Meth., 212, 319

Hofmann, W., Jung, I., Konopelko, A., et al. 1999, Astropart. Phys., 12, 135

Hwang, U., \& Gotthelf, E. V. 1997, ApJ, 475, 665

Hwang, U., Hughes, J. P., \& Petre, R. 1998, ApJ, 497, 833

Hughes, J. P. 2000, ApJ, in press [astro-ph/0010122]

Katz-Stone, D. M., Kassim, N. E., Joseph, T., et al. 2000, AJ, 529,453

Kirk, J. G., \& Dendy, R. O. 2000, J. Phys. G: Nucl. Part. Phys., in press 
Konopelko, A., Hemberger, M., Aharonian, F. A., et al. 1999, Astropart. Phys., 10, 275

Koyama, K., Petre, R., Goffhelf, E. V., et al. 1995, Nature, 378,255

Koyama, K., Kinusaga, K., Matsuzaki, K., et al. 1997, PASJ, 49, L7

Krennrich, F., et al. 1999, Towards a Major Atmospheric Cherenkov Detector-VI, ed. B. L. Dingus, M. H. Salamon, \& D. B. Kieda (AIP Conf. Ser. 515, New York, 2000), 515

Laming, J. M. ApJ, 499, 309

Li, T., \& Ma, Y. 1983, ApJ, 272, 317

Longair, M. S. High Energy Astrophysics (Cambridge University Press, 1997)

Lorenz, E., et al. 1999, Towards a Major Atmospheric Cherenkov Detector-VI, ed. B. L. Dingus, M. H. Salamon, \& D. B. Kieda (AIP Conf. Ser. 515, New York, 2000), 510

Muraishi, H., Tanimori, T., Yanagita, S., et al. 2000, A\&A, $354, \mathrm{~L} 57$

Naito, T., \& Takahara, F. 1994, J. Phys G: Nucl. Part. Phys., 20,477

Petre, R., Allen, G. E., \& Hwang, U. 1999, Astronomische Nachrichten, 320, 199

Prahl, J., \& Prosch, C. for the HEGRA Collab. 1997, Proc. 25th Int. Cosmic Ray Conf. (Durban) 3, 217
Prosch, C., Feigl, E., Plaga, R., et al. 1996, A\&A, 314, 275

Pühlhofer, G., Völk, H., Wiedner, C. A., et al. 1999, Proc. 26th Int. Cosmic Ray Conf. (Salt Lake City) 3, 492

Reynolds, S. P., \& Keohane, J. W. 1999, ApJ, 525, 368

Reynoso, E. M., Velazquez, P. F., Dubner, G. M., \& Goss, W. M. 1999, AJ, 117, 1827

Reynoso, E. M., Moffett, D. A., Goss, W. M., et al. 1997, ApJ, 491, 816

Rowell, G. P., Naito, T., Dazeley, S. A., et al. 2000, A\&A, 359, 337

Schwarz, U. J., Goss, W. M., Kalberla, P. M., \& Benaglia, P. 1995, A\&A, 299, 193

Seward, F., Gorenstein, P., \& Tucker, W. 1983, ApJ, 266, 287

Smith, A., Davelaar, J., Peacock, A., et al. 1988, ApJ, 325, 288

Tan, S. M., \& Gull, S. F. 1985, MNRAS, 216, 949

Tanimori, T., Hayami, Y., Kamei, S., et al. 1998, ApJ, 492, L33

Tatischeff, V., Ramaty, R., \& Kozlovsky, B. 1998, ApJ, 504, 874

Velazquez, P. F., Gomez, D. O., Dubner, G. M., et al. 1998, A\&A, 334, 1060

Völk, H. J. 1997 in Towards a Major Atmopsheric Cerenkov Detector-V, ed. O. C. deJager (WESPRINT, Potchefstroom 1997), 87 